\title{
Evolutionary conserved longevity genes and human cognitive abilities in elderly cohorts
}

\author{
Lorna M Lopez ${ }^{\star 1,2,14}$, Sarah E Harris ${ }^{2,3}$, Michelle Luciano ${ }^{1,2}$, Dave Liewald ${ }^{2}$, Gail Davies ${ }^{1}$, Alan J Gow ${ }^{1,2}$, \\ Albert Tenesa $^{4,5}$, Antony Payton ${ }^{6}$, Xiayi Ke ${ }^{6,7}$, Lawrence J Whalley ${ }^{8}$, Helen Fox ${ }^{9}$, Paul Haggerty ${ }^{10}$, \\ William Ollier $^{6}$, Andrew Pickles ${ }^{11}$, David J Porteous ${ }^{2,3}$, Michael A Horan ${ }^{12}$, Neil Pendleton ${ }^{12}$, John M Starr ${ }^{1,13}$ \\ and Ian J Deary ${ }^{1,2}$
}

Genetic influences have an important role in the ageing process. The genetic factors that influence success in bodily ageing may also contribute to the successful ageing of cognitive abilities. A comparative genomics approach found longevity genes conserved between yeast Saccharomyces cerevisiae and nematode Caenorhabditis elegans. We hypothesised that these longevity genes influence variance in cognitive ability and age-related cognitive decline in humans. Here, we investigated six of these genes that have human orthologs and show expression in the brain. We tested AFG3L2 (MIM: 604581, AFG3 ATPase family gene 3-like 2 (yeast)), FRAP1 (MIM: 601231, a FK506 binding protein 12-rapamycin associated protein), MAT1A, MAT2A (MIM: 610550 and 601468, methionine adenosyltransferases I alpha and II alpha, respectively), SYNJ1 and SYNJ2 (MIM: 604297 and 609410, synaptojanin-1 and synaptojanin-2, respectively) in approximately 1000 healthy older Scots: the Lothian Birth Cohort 1936 (LBC1936). They were tested on general cognitive ability at age 11 years. At a mean age of 70 years, they re-sat the same general cognitive ability test and underwent an additional battery of diverse cognitive tests. In all, 70 tag and functional SNPs in the six longevity genes were genotyped and tested for association with cognition and cognitive ageing in LBC1936. Suggestive associations were detected between SNPs in SYNJ2, MAT1A, AFG3L2 and SYNJ1 and a general memory factor and general cognitive ability at age 11 and 70 years. Replication studies for cognitive ability associations were performed in 2506 samples from the Cognitive Ageing Genetics in England and Scotland consortium. A meta-analysis replicated the SYNJ2 association with cognitive abilities (lowest $P=0.00077$ ). SYNJ2 is a novel gene in which variation is potentially associated with cognitive abilities.

European Journal of Human Genetics (2012) 20, 341-347; doi:10.1038/ejhg.2011.201; published online 2 November 2011

Keywords: cognition; cognitive ageing; longevity genes; gene association; SYNJ2

\section{INTRODUCTION}

With the growing absolute numbers and proportions of older people in societies, the changes wrought by ageing have become a research priority. ${ }^{1}$ Very high on this list is the problem of cognitive ageing. Cognitive ageing is a much-feared aspect of growing old and is the major cause of older people's losing independence and lowering their quality of life. ${ }^{2}$ There are marked individual differences in agerelated cognitive changes, ${ }^{3,4}$ and among the causes of this variation are genetic, medical, psychological, and social and lifestyle factors. ${ }^{5,6}$ Beyond APOE, and excluding the dementias, there are no solid associations between genetic variants and cognitive functions in old age, despite many suggestions and replication attempts. ${ }^{7}$

There are well-established phenotypic associations between cognition, health and longevity. ${ }^{8,9}$ The 'common-cause' hypothesis of cognitive ageing states that there are general bodily factors that affect both physical and mental changes with age. ${ }^{10}$ Human longevity has been associated with higher cognitive abilities, ${ }^{11}$ even when intelligence is measured in childhood or early adulthood and the assessment of survival has been conducted several decades later. ${ }^{12,13}$ Behavioural genetic studies have shown that genetic factors influence both longevity ${ }^{14}$ and cognitive traits, ${ }^{7}$ alongside other stochastic variation. ${ }^{15}$ Given the well-replicated phenotypic association between cognitive abilities and longevity, it is plausible that part of this is explained by shared genetic factors (genetic correlation); therefore, to explore this, it is useful to examine genes involved in longevity for their association with cognitive abilities and cognitive ageing. We can study this cognitive function and lifetime cognitive change in older people as we have the advantage of having an ageing sample with a measure of cognitive ability from youth.

In an effort to harness the 'new biology of ageing research, ${ }^{16}$ we chose evolutionary-conserved, longevity genes uncovered by a

${ }^{1}$ Department of Psychology, The University of Edinburgh, Edinburgh, UK; ${ }^{2}$ Centre for Cognitive Ageing and Cognitive Epidemiology, The University of Edinburgh, Edinburgh, UK; ${ }^{3}$ Medical Genetics Section, The University of Edinburgh, Edinburgh, UK; ${ }^{4}$ MRC Human Genetics Unit, Institute of Genetics and Molecular Medicine, Edinburgh, UK; ${ }^{5}$ The Roslin Institute, Royal (Dick) School of Veterinary Studies, University of Edinburgh, Roslin, UK; ${ }^{6}$ Centre for Integrated Genomic Medical Research, School of Cancer and Enabling Sciences, The University of Manchester, Manchester, UK; ${ }^{7}$ MRC Centre of Epidemiology for Child Health, UCL Institute of Child Health, London, UK; ${ }^{8}$ University of Aberdeen, Institute of Applied Health Sciences, Foresterhill, Aberdeen, UK; ${ }^{9}$ Scottish Dementia Research Network, Royal Victoria Hospital, Edinburgh, UK; ${ }^{10}$ Rowett Institute of Nutrition and Health, University of Aberdeen, Aberdeen, UK; ${ }^{11}$ Department of Biostatistics, Institute of Psychiatry, King's College, London, UK; ${ }^{12}$ Mental Health and Neurodegeneration, School of Community Based Medicine, University of Manchester, Salford Royal NHS Trust, UK; ${ }^{13}$ Geriatric Medicine Unit, The University of Edinburgh, Royal Victoria, Edinburgh, UK *Correspondence: Dr LM Lopez, Department of Psychology, The University of Edinburgh, 7 George Square, Edinburgh EH8 9JZ, UK. Tel: +44 (0)13 1650 8495;

E-mail: lorna.lopez@ed.ac.uk

14Lorna M Lopez has previously published under her maiden name Lorna M Houlihan.

Received 11 March 2011; revised 25 July 2011; accepted 6 September 2011; published online 2 November 2011 
comparative functional genomics investigation on ageing between two divergent eukaryotic species, the yeast Saccharomyces cerevisiae and the nematode Caenorhabditis elegans. ${ }^{17}$ The investigators had reported the replicative lifespan phenotypes (the number of times that a mother cell can bud to form a daughter) for single-gene deletions of the yeast orthologs of worm ageing genes. They identified 25 genes that modulate yeast replicative lifespan and suggested that 'many of these genes, and the pathways within which they function, are likely to modulate ageing in mammals, and that mammalian orthologs of these gene pairs are reasonable candidates as potential therapeutic targets for age-associated diseases' (Smith et al, ${ }^{17}$ 568). From this list of 25 genes, we narrowed our focus to brain-expressed ${ }^{18}$ genes in humans, resulting in six genes - AFG3L2 (MIM: 604581, AFG3 ATPase family gene 3-like 2 (yeast)), FRAP1 (MIM: 601231, a FK506 binding protein 12-rapamycin-associated protein), MAT1A, MAT2A (MIM: 610550 and 601468, methionine adenosyltransferases I alpha and II alpha, respectively), SYNJ1 and SYNJ2 (MIM: 604297 and 609410, synaptojanin-1 and synaptojanin-2, respectively). These genes are novel candidate genes for cognition and do not fall in any of the linkage regions for cognitive traits. $^{7}$ We proposed that variation in these evolutionary conserved genes for longevity may be associated with individual differences in human cognitive ageing. We tested this hypothesis in a Scottish cohort, assessed for cognitive ability at age 11 and 70 years, by genotyping haplotype-tagging genetic variants in the six genes. We sought replication in newly available genome-wide association genotype data from cohorts of older individuals from England and Scotland in the Cognitive Ageing Genetics in England and Scotland (CAGES) consortium: the Lothian Birth Cohort of 1921 (LBC1921), ${ }^{19}$ the Aberdeen Birth Cohort of 1936 (ABC1936) ${ }^{19,20}$ and the Manchester and Newcastle Longitudinal Studies of Cognitive Ageing. ${ }^{21}$

\section{MATERIALS AND METHODS}

\section{Subjects}

There are 1091 individuals ( 543 females) in the LBC1936. All were born in 1936 and attended school in Scotland in 1947. At an average age of 11 years, they took a valid IQ-type test - a version of the Moray House Test No. 12 (MHT) in the nationwide Scottish Mental Survey 1947 (SMS1947; $N=70805) .{ }^{22}$ At age $\sim 70$ years, the LBC1936 were recruited as surviving and still relatively healthy participants of the SMS1947 who were living in Edinburgh and the surrounding areas (Lothians) of Scotland. They re-sat the same mental test and other cognitive and medical tests, as described elsewhere in detail. ${ }^{23}$ All participants in the study lived independently in the community and travelled to the Wellcome Trust Clinical Research Facility (WTCRF) at the Western General Hospital, Edinburgh, UK for testing. DNA samples were available for 1078 participants. In all, 12 participants were excluded owing to possible dementia: 11 scored $<24$ on the Mini-Mental State Examination (MMSE) ${ }^{24,25}$ and one had an incomplete MMSE test. The final sample with phenotype data used in the analyses was 1038 individuals (521 females), with a mean age of 69.5 years (range 67.6-71.3). They had a mean age of 10.9 years (range 10.4-11.4) when tested in the SMS1947.

The replication cohorts were from the CAGES project: the LBC1921 ( $N=517$ (303 females), mean age 79.1 years $(S D=0.6)),{ }^{19}$ the $\mathrm{ABC} 1936(N=426,(208$ females), mean age 64.4 years $(\mathrm{SD}=0.9))^{19,20}$ and the Manchester and Newcastle Longitudinal Studies of Cognitive Ageing (Manchester N=805 (572 females), Newcastle $N=758$ (536 females), median age of 65 years, range 44-93 years), ${ }^{21}$ as described previously. ${ }^{26}$ All four cohorts comprised nonclinical samples of relatively healthy people from middle to older adulthood.

\section{Cognitive tests}

A full description of the cognitive tests applied to LBC1936 is available elsewhere. ${ }^{23}$ The tests pertinent to this study are described in brief below. A general measure of cognitive ability with an emphasis on verbal reasoning (MHT) was administered when participants were a mean age of 11 years in the
SMS1947 (Scottish Council for Research in Education). ${ }^{22}$ The MHT was re-administered at a mean age of almost 70 years for LBC1936, using the same instructions and 45 minute time limit that were applied at age 11 years. Cognitive tests assessing reasoning and different aspects of memory were administered to the LBC1936 at age 70 years. These include: logical memory, backward digit span, spatial span and verbal paired associates from the Wechsler Memory Scale-III ${ }^{\mathrm{UK}},{ }^{27}$ letter-number sequencing, matrix reasoning and block design from the WAIS-III ${ }^{\mathrm{UK}} ;{ }^{27}$ The information processing speed battery comprised two psychometric tests from the WAIS-III ${ }^{\mathrm{UK}}$ (digit symbol and symbol search) and two elementary cognitive tasks: simple and four choice reaction time (RT), and inspection time, which is a psychophysical assessment of the efficiency of the early stages of visual decision-making. ${ }^{19,28}$

The replication was performed in four cohorts from the CAGES study: LBC1921, ${ }^{19}$ ABC1936 ${ }^{19,20}$ and the Manchester and Newcastle Longitudinal Studies of Cognitive Ageing, ${ }^{21}$ previously described in detail. Individuals with MMSE <24 were removed from LBC1921 and ABC1936. In LBC1921, scores for the MHT taken at age 11 and 79 years, logical memory, Raven's Progressive Matrices and verbal fluency, all taken at age 79 years were available. In ABC1936, scores for the MHT taken at age 11 years, block design, auditory verbal learning test (AVLT), and Raven's Progressive Matrices, digit symbol and Uses of Common Objects taken at age 64 years were used. For Manchester and Newcastle, the cognitive tests applied were described previously. ${ }^{21,26}$

\section{SNP selection}

Tagging SNPs were selected to tag haplotypes from the specific gene regions and $5 \mathrm{~kb}$ either side of the gene. Genotype data were downloaded from the HapMap CEPH population (Release 22). The tagging SNPs were chosen by Tagger $^{29}$ in Haploview v. $4.1^{30}$ using the pairwise tagging method with the default settings $\left(r^{2}=0.8\right.$ ), with one exception (minimum minor allele frequency (MAF) 0.05). In all, 70 tag SNPs act as direct proxies to all other SNPs in the six genes because they are highly correlated with one another $\left(r^{2} \geq 0.8\right)$. Coding non-synonymous SNPs were also chosen (rs2502601 in SYNJ2 and rs2254562 in SYNJ1) as the allele changes $(\mathrm{A} \rightarrow \mathrm{G})$ in both SNPs cause missense mutations and residue changes (Glu $\rightarrow$ Gly, Lys $\rightarrow$ Arg, respectively). These two SNPs predict important functionality of the SYNJ2 and SYNJ1 genes, and consequently are informative as potential causative variants. In total, 70 SNPs were genotyped. This SNP selection was carried out before genome-wide SNP data becoming available on the LBC1936 sample.

Conventionally significantly associated SNPs $(P<0.05)$ were chosen for replication for cognitive abilities in the CAGES cohort: $N=21$ SNPs. The SNPs were extracted from genome-wide data as described previously ${ }^{26}$ and genotype data were imputed to HapMap phase II CEU data as the reference sample, using NCBI build 36 (UCSC hg18) in the MACH software. ${ }^{31}$ Before imputation, SNPs were removed that diverged from Hardy-Weinberg equilibrium (HWE) with a significance $P<1 \times 10^{-3}$ and SNPs with an MAF $<0.01 .^{32}$

\section{Genotyping}

Genomic DNA was isolated from whole blood by standard procedure at the WTCRF Genetics Core, Western General Hospital, Edinburgh. In total, 49 markers were genotyped using a competitive allele-specific PCR system (KASPar) by KBiosciences (Herts, UK). SYNJ2 was not fully covered in the first SNP selection owing to a genome browser change and human error. To rectify this, 21 SNPs in SYNJ2 (rs1750043-rs13217929) were extracted from a whole genome scan on LBC1936 as described previously. ${ }^{33}$ The 'force-include' option in Tagger was used to force the inclusion of the SNPs genotyped in the whole genome scan as tagging SNPs, and the same level of tagging coverage for SYNJ2 $\left(r^{2} \geq 0.8\right)$ was achieved.

\section{Statistical analyses}

Cognitive phenotypes. The cognition data were prepared by removing outliers in the cognitive variable data with $Z$ scores greater than \pm 3 . A general cognitive ability factor was derived from principal components analysis of one WMS-III subtest (digit-span backwards) and five WAIS-III subtests (matrix reasoning, letter-number sequencing, block design, symbol search and digit symbol), as described previously. ${ }^{34}$ A general speed factor was separately derived from principal components analysis of speed measures (choice RT 
mean, simple RT mean (log-transformed), digit symbol, inspection time and symbol search). ${ }^{34}$ A general memory factor was derived from principal components analysis of WMS III logical memory I total recall score $(A+B+B 2)$, WMS III logical memory II delayed recall total score $(\mathrm{A}+\mathrm{B})$, WMS III spatial span forward, WMS III spatial span backward, WMS III verbal paired associates I (List $\mathrm{A}+\mathrm{B}+\mathrm{C}+\mathrm{D}$ ), WMS III verbal paired associates II recall total score, WAIS III letter-number sequencing and WAIS III digit-span backwards. ${ }^{35}$ Outliers were removed before principal components analysis. The MHT1947 score at age 11 years was residualised for age. The sample size, the mean values and standard deviations of the cognitive variables have been published previously. ${ }^{36}$ This preparation was performed using SPSS version 14.0.

For the replication cohorts, similar preparation of the cognitive phenotypes was performed. Outliers with $Z$ scores greater than \pm 3 were removed. Phenotypes were standardised to enable comparison. In LBC1921, a general cognitive ability factor was derived from principal components analysis of Raven's Progressive Matrices, verbal fluency and logical memory as described previously. ${ }^{35}$ In ABC1936, a general cognitive ability factor was derived from principal components analysis of Raven's Progressive Matrices, Digit Symbol, AVLT, Uses of Common Objects as described previously as $g_{\mathrm{f}}{ }^{26}$ In LBC1921 and $\mathrm{ABC} 1936$, the MHT score at age 11 years was residualised for age in days at age 11 years. The phenotypes in LBC1921 and ABC1936 were corrected for age and gender and the standardised scores were used for all subsequent analyses.

In Manchester and Newcastle samples, the individual cognitive tests have been described previously, ${ }^{22}$ and the phenotypes for this study of cognition in old age were prepared together as follows. For a general cognitive ability factor, empirical Bayes' (EB) estimates for each individual were obtained from a random effects model fitted by maximum likelihood (ML) to the standardised age regressed residuals obtained for each sex from the Alice Heim 4 test (1970) and the Cattell (1960) 'Culture Fair' test scores. ${ }^{26}$ A similar approach was taken for a speed factor based on the Visual Search for letters and Savage (1984) Alphabet Coding Task tests. With up to seven measures (Verbal Free Recall for 30 words, Verbal Free Recall for 10 words, Cumulative Verbal Learning, Pictorial Recognition Memory test, Memory for Shapes and Location, Propositions about people, Memory Circle) available to form a general memory factor, individual EB estimates were obtained from the standardised age regressed residuals from each test using a one-factor model fitted by ML.

Although different sets of tests were used to construct the general cognitive ability factor, it is well established that the general factors derived from different mental test batteries tend to rank people almost identically. ${ }^{37}$

Genotype data. All SNPs were in HWE as judged by the HW exact SNP tests (all $P$-values $>0.001$ (Haploview default) and are reported in Supplementary Table 1). The genotyping data were of good quality, as the mean genotyping rate in LBC1936 was 99\% (range 92-100\%) in 1038 samples. The MAF of all markers were $>0.044$. The genotype frequencies were similar to the HapMap $\mathrm{CEPH}$ population (mean difference in genotype frequencies $=0.03$, minimum 0.002 , maximum 0.08). Further characteristics of the SNPs investigated are listed in Supplementary Table 1. SNPs in each replication sample were checked for MAF (all markers MAF $>0.08$ ), HWE (all $P$-values $>0.001$ ) and imputation quality (mean $r^{2}=0.97(0.053)$ ).

Association analysis. In the discovery sample, LBC1936, genotype-phenotype analyses were performed using PLINK version 1.07. ${ }^{38}$ Linear regression analysis under an additive genetic model was performed, including gender and age in days at testing at age 70 years as covariates. In a separate analysis, MHT score at age 11 years (age residualised) was included as a covariate to adjust for previous cognitive ability, thus allowing us to specifically identify associations with cognitive functions in old age, while adjusting for cognitive differences in youth (ie, cognitive ageing). This inclusion of a previous measurement as a covariate is a widely used 'measure' of change. Standardised $\beta$ scores, the standard error (SE) and their corresponding $P$-values are reported.

In the replication sample, linear regression analysis for an additive genetic model was performed using MACH2QTL, ${ }^{39}$ incorporating dosage information. For ABC1936, age and sex were included as covariates in the model. For Manchester and Newcastle replication cohorts, the analysis was performed separately by gender owing to the preparation of the phenotypes in a sex-specific manner. $\beta$ Scores, the $\mathrm{SE}$ and the corresponding $P$-values are reported. Meta-analyses were performed in Manchester and Newcastle cohorts to combine results for men and women, and in all the cohorts on overlapping phenotypes (General Cognition Factor and Memory tests) in the replication samples using an inverse variance weighted model. ${ }^{40}$

Statistical significance and power. In the first instance, the significance threshold was determined by the Bonferroni method, which corrects the critical significance level by the number of tests $(n)$ performed $(\alpha=0.05 / n)$, and is commonly used in candidate gene studies. ${ }^{41}$ It is recognized that the Bonferroni correction can be overly conservative for non-independent tests. ${ }^{42}$ Therefore, we calculated the number of independent tests performed. Based on matrix spectral decomposition, ${ }^{43}$ the 70 SNPs represent 46 independent variables. A principal component analysis of the specific cognitive test components of the five cognitive measures in the discovery sample of LBC1936 was performed. These are the Moray House test at age 11 years, Moray House test at age 70 years, logical memory I total recall score, logical memory II delayed recall total score, spatial span forward, spatial span backward, verbal paired associates I and verbal paired associates II, symbol search, digit symbol, simple RT mean (log-transformed), choice RT mean, inspection time, matrix reasoning, letternumber sequencing, digit-span backwards and block design. This resulted in three components with eigenvalues $>1(5.04,1.47,1.15)$. It should be noted, however, that the scree plot (available from the first author upon request) confirms the presence of one general factor of cognition, accounting for $36 \%$ of the total variance. The mean of the absolute factor loadings on the first unrotated component was 0.59 (range from 0.37 for simple RT mean to 0.80 for the Moray House Test at age 70 years). The adjusted Bonferroni significance threshold thus applied was $P<0.0004 \quad(0.05 /(3$ cognitive components $\times 46$ independent SNPs)). Replication was sought for nominal significant associations $(P<0.05)$. For evidence of replication, $P<0.05$ was taken as significant evidence.

The power to detect an additive effect of a causal variant, in linkage disequilibrium $D^{\prime}=1$, of a marker with an allele frequency of 0.2 , accounting for $1-2 \%$ of the variance, at type- 1 error rate adjusted for multiple testing ( $P$-value $\leq 0.0004)$ was $28-77 \%$ in LBC1936 $(N=1038)$. This was estimated using the variance component quantitative trait loci association module in the genetic power calculator. ${ }^{44}$

\section{RESULTS}

In all, 70 haplotype-tagging SNPs in six genes (AFG3L2, FRAP1, MAT1A, MAT2A, SYNJ1 and SYNJ2) were tested for association with cognitive abilities, including age and gender as covariates. The cognitive abilities tested were a verbal reasoning test at age 11 years (a version of the MHT), the same test again at age 70 years, a general cognitive ability factor, a general memory factor and a general mental speed factor, also at age 70 years. No SNP associations surpassed the Bonferroni level of correction for multiple testing $(P<0.0004)$. Suggestive single SNP associations (unadjusted $P$-values $<0.05$ ) were detected in variants of four of these genes with cognitive abilities: SYNJ2, MAT1A, AFG3L2 and SYNJ1 (Table 1). No associations with cognition were detected in FRAP1 and MAT2A (all $P$-values $>0.1$ ) (Table 1). Supplementary Figure 1 shows that 14 of the 44 SNPs tagging SYNJ2 were suggestively associated with cognitive abilities. The strongest association was an intronic SYNJ2 SNP rs10945973 with the general memory factor $(P=0.004)$.

Variants in these six genes (AFG3L2, FRAP1, MAT1A, MAT2A, SYNJ1 and SYNJ2) were further tested for association with cognitive ageing by including age 11 years cognitive ability (based on MHT scores), in addition to sex and age, as a covariate. Significant SNP associations were detected in four of the genes (13 SNPs): SYNJ2, MAT1A, AFG3L2 and SYNJ1. No associations with cognitive ageing were detected in FRAP1 and MAT2A (all P-values >0.1) (Supplementary Table 2). 
Table 1 Significant associations of longevity gene variants to cognitive abilities in LBC1936

\begin{tabular}{|c|c|c|c|c|c|c|c|c|c|c|c|c|c|c|c|c|c|c|}
\hline Gene & $\mathrm{CHR}$ & SNP & $M A$ & \multicolumn{3}{|c|}{ MHT age 11 years } & \multicolumn{3}{|c|}{ MHT age 70 years } & \multicolumn{3}{|c|}{ G cognition } & \multicolumn{3}{|c|}{ G memory } & \multicolumn{3}{|c|}{ G speed } \\
\hline \multirow[t]{9}{*}{ SYNJ2 } & 6 & rs11961283 & C & -0.064 & 0.032 & 0.043 & -0.043 & 0.031 & 0.158 & -0.022 & 0.030 & 0.467 & -0.071 & 0.031 & 0.022 & -0.023 & 0.032 & 0.472 \\
\hline & 6 & rs7772395 & C & -0.068 & 0.032 & 0.035 & -0.051 & 0.031 & 0.103 & -0.041 & 0.031 & 0.190 & -0.059 & 0.031 & 0.059 & -0.053 & 0.032 & 0.104 \\
\hline & 6 & rs10455935 & A & -0.037 & 0.032 & 0.251 & -0.028 & 0.031 & 0.378 & -0.017 & 0.031 & 0.582 & -0.063 & 0.031 & 0.043 & 0.010 & 0.032 & 0.752 \\
\hline & 6 & rs10945973 & A & 0.016 & 0.032 & 0.623 & 0.036 & 0.031 & 0.253 & 0.036 & 0.031 & 0.238 & 0.090 & 0.031 & 0.0037 & 0.000 & 0.032 & 0.998 \\
\hline & 6 & rs9456954 & A & -0.042 & 0.032 & 0.187 & -0.070 & 0.031 & 0.024 & 0.010 & 0.031 & 0.734 & -0.042 & 0.031 & 0.169 & -0.026 & 0.032 & 0.416 \\
\hline & 6 & rs7758206 & C & -0.050 & 0.032 & 0.117 & -0.063 & 0.031 & 0.040 & -0.008 & 0.030 & 0.784 & -0.038 & 0.031 & 0.213 & -0.047 & 0.032 & 0.139 \\
\hline & 6 & rs9459093 & C & -0.034 & 0.032 & 0.283 & -0.059 & 0.031 & 0.058 & -0.043 & 0.031 & 0.161 & -0.079 & 0.031 & 0.011 & 0.007 & 0.032 & 0.822 \\
\hline & 6 & rs751873 & $\mathrm{T}$ & -0.030 & 0.033 & 0.366 & -0.023 & 0.032 & 0.475 & 0.000 & 0.031 & 0.995 & -0.067 & 0.032 & 0.033 & 0.033 & 0.033 & 0.310 \\
\hline & 6 & rs3818457 & A & 0.067 & 0.033 & 0.043 & 0.034 & 0.032 & 0.288 & 0.041 & 0.031 & 0.193 & 0.063 & 0.032 & 0.047 & 0.024 & 0.033 & 0.460 \\
\hline \multirow[t]{3}{*}{ SYNJ1 } & 21 & rs845022 & $T$ & -0.058 & 0.032 & 0.069 & -0.036 & 0.031 & 0.250 & -0.068 & 0.030 & 0.025 & -0.033 & 0.031 & 0.285 & -0.047 & 0.032 & 0.138 \\
\hline & 21 & rs7279487 & C & 0.090 & 0.032 & 0.0050 & 0.064 & 0.031 & 0.039 & 0.060 & 0.031 & 0.051 & 0.059 & 0.031 & 0.055 & 0.064 & 0.032 & 0.045 \\
\hline & 21 & rs844996 & G & 0.086 & 0.032 & 0.0076 & 0.047 & 0.031 & 0.135 & 0.067 & 0.031 & 0.031 & 0.063 & 0.031 & 0.046 & 0.059 & 0.032 & 0.067 \\
\hline
\end{tabular}

Abbreviations: MHT, Moray House Test, which is a verbal reasoning test taken at age 11 and 70 years; G, general ability factors; $\mathrm{CHR}$, chromosome; MA, minor allele; $P, P$-value; $\beta$, the standardised regression coefficient, where a positive regression coefficient shows that the minor allele increases phenotype mean.

Associations that surpass the nominal significance level adjusted for the number of independent phenotypes tested are shown ( $P<0.05$, in bold). The 50 SNPs that did not show association with cognitive abilities are not shown; these latter results are available from the authors.

The $P$-values are represented in italics.
Thive

Overall, 21 SNPs in four genes (SYNJ2, MAT1A, AFG3L2 and SYNJ1) were suggestively associated (unadjusted $P<0.05$ ) with cognitive abilities in old age (not cognitive ageing) in our discovery cohort, LBC1936, shown in Table 1 and Supplementary Table 2. These 21 SNPs explain $0.36-0.8 \%$ (by squaring the standardised $\beta$ values in Table 1) of the variance in the cognitive phenotypes investigated. Replication of the 21 SNPs associated with cognitive abilities was sought in four elderly independent cohorts from the CAGES consortium with relevant cognitive phenotypes and genotype data imputed from a genome-wide association study. Independent association analysis was performed in each replication cohort. In ABC1936, MHT at age 11 years, block design (to replicate MHT at age 70 years), general cognitive factor and AVLT (to replicate memory) were tested for association. In LBC1921, MHT at age 11 years, MHT at age 79 years, general cognitive factor and logical memory (to replicate memory) were tested for association. In Manchester and Newcastle, general cognitive, speed and memory factors were tested for association.

Replication of SNP associations with SYNJ2 was present in the four cohorts: ABC1936, LBC1921, Manchester and Newcastle (Supplementary Tables 3-6). Some of the SNP associations were replicated in the targeted phenotype from LBC1936 (LBC1921: rs6455937 and rs9459093 for memory, and rs7772395 for MHT age 11 years; Newcastle: rs6455937 and rs10455935 for memory). Across all the replication samples, the effect was in the same direction for the significant SNPs, and it was the same direction as the discovery sample, LBC1936. A meta-analysis showed significant association of six SYNJ2 SNPs (Table 2). Three SNPs were significant for both general cognitive ability and memory (rs6455937, rs7772395 and rs10455935). Three SNPs were significant for memory abilities only: rs11961283, rs10945973 and rs9459093. These six SNPs explain $0.45-1.4 \%$ of the variance in the meta-analysed general cognitive ability factor and memory ability.

\section{DISCUSSION}

This study proposed six novel candidate genes (AFG3L2, FRAP1, MAT1A, MAT2A, SYNJ1 and SYNJ2) for cognition in old age and cognitive ageing. The novelty of the choice was to select evolutionary conserved genes for longevity, found through a comparative functional genomics approach and to test for association with human cognitive ageing. Initially, we investigated 70 haplotype-tagging SNPs in six genes for association with cognitive ability at age 11 and 70 years. We tested for association with cognitive ageing by including cognitive ability at age 11 years as a covariate. There were suggestive associations in four genes with cognitive ability, SYNJ2, MAT1A, AFG3L2 and SYNJ1. The association of 13 out of the 20 suggestive SNPs for cognitive ability remained associated with cognitive ageing, with the same direction of effect, but to a lesser degree of effect and significance as shown in Table 1 and Supplementary Table 2. This could possibly be explained by the phenotype as any change variable is always less reliably measured than a trait, or that the genetic associations are not specific to cognitive ageing. SNP associations in SYNJ2 were replicated in a meta-analysis of general cognitive ability and memory ability. Given the neuronal functionality of SYNJ2, this gene may be one of the many genes with a small effect influential in cognitive abilities.

SYNJ2 has biological plausibility to support its role in cognitive processes. SYNJ2 is an ubiquitously expressed inositol polyphosphate 5-phosphatase, shown specifically to be expressed in nerve terminals ${ }^{45}$ and differentially expressed in hippocampal subregions of the 
Table 2 Meta-analysis of $G$ factor $(N=2401)$ and memory $(N=2412)$ in the replication samples ABC1936, LBC1921, Manchester and Newcastle

\begin{tabular}{|c|c|c|c|c|c|c|c|c|c|c|c|}
\hline \multirow[b]{2}{*}{ Gene } & \multirow[b]{2}{*}{$E A$} & \multirow[b]{2}{*}{$O A$} & \multirow[b]{2}{*}{$M A$} & \multicolumn{4}{|c|}{ G cognition } & \multicolumn{4}{|c|}{ Memory } \\
\hline & & & & Effect & $S E$ & $P$ & Dir & Effect & $S E$ & $P$ & Dir \\
\hline \multicolumn{12}{|l|}{ SYNJ2 } \\
\hline rs11961283 & $\mathrm{T}$ & C & C & 0.067 & 0.035 & 0.056 & +---++ & 0.103 & 0.034 & 0.0025 & ++++++ \\
\hline rs6455937 & $A$ & C & C & 0.078 & 0.030 & 0.0090 & ++++-+ & 0.089 & 0.029 & 0.0023 & +++-++ \\
\hline rs7772395 & $A$ & C & C & 0.098 & 0.036 & 0.0066 & +++-++ & 0.116 & 0.035 & 0.0009 & +++-++ \\
\hline rs10455935 & $A$ & G & $A$ & -0.065 & 0.029 & 0.028 & ------ & -0.085 & 0.029 & 0.0032 & ---+-- \\
\hline rs10455936 & $\mathrm{T}$ & $\mathrm{C}$ & $\mathrm{T}$ & 0.001 & 0.034 & 0.977 & +---++ & -0.008 & 0.033 & 0.806 & +--+-+ \\
\hline rs10945973 & $A$ & G & $A$ & 0.042 & 0.030 & 0.159 & ++++++ & 0.066 & 0.029 & 0.024 & +++-++ \\
\hline rs6906464 & $A$ & $T$ & $T$ & 0.042 & 0.034 & 0.220 & +-+-++ & 0.063 & 0.033 & 0.059 & +++-++ \\
\hline rs9356200 & $\mathrm{T}$ & C & C & -0.027 & 0.029 & 0.364 & ++--+- & -0.051 & 0.029 & 0.078 & ------ \\
\hline rs9456954 & $A$ & $\mathrm{~T}$ & A & -0.006 & 0.032 & 0.854 & -+-+-- & -0.054 & 0.032 & 0.093 & ------ \\
\hline rs7758206 & C & G & C & 0.009 & 0.037 & 0.814 & ++-+-- & -0.051 & 0.036 & 0.158 & +---++ \\
\hline rs9459093 & $\mathrm{T}$ & C & C & 0.028 & 0.029 & 0.332 & +-++-+ & 0.058 & 0.029 & 0.042 & +-++-+ \\
\hline rs751873 & $\mathrm{T}$ & C & $\mathrm{T}$ & 0.052 & 0.031 & 0.087 & -+-+-+ & -0.002 & 0.030 & 0.951 & +----+ \\
\hline rs3818457 & $\mathrm{T}$ & C & $A(T)$ & 0.023 & 0.029 & 0.421 & +-++-- & 0.021 & 0.028 & 0.463 & +-+-+- \\
\hline rs1744169 & $A$ & G & $T(A)$ & 0.021 & 0.030 & 0.478 & +-++-+ & 0.013 & 0.029 & 0.648 & +++-++ \\
\hline rs2502601 & $A$ & $\mathrm{G}$ & $\mathrm{T}(\mathrm{A})$ & 0.009 & 0.029 & 0.768 & +--++- & 0.007 & 0.028 & 0.816 & +-+-++ \\
\hline $\begin{array}{l}\text { MAT1A } \\
\quad \text { rs3851059 }\end{array}$ & $A$ & G & $A$ & -0.025 & 0.031 & 0.430 & ---+-+ & -0.013 & 0.031 & 0.679 & +-++-- \\
\hline rs4933327 & $A$ & G & A & -0.023 & 0.035 & 0.511 & --++-- & -0.030 & 0.034 & 0.380 & +-++-- \\
\hline $\begin{array}{l}\text { AFG3L2 } \\
\text { rs9964979 }\end{array}$ & $\mathrm{T}$ & C & $\mathrm{T}$ & -0.019 & 0.040 & 0.635 & ---+++ & -0.032 & 0.039 & 0.407 & +---+- \\
\hline \multicolumn{12}{|l|}{ SYNJ1 } \\
\hline rs845022 & $A$ & $\mathrm{~T}$ & $\mathrm{~T}$ & -0.027 & 0.029 & 0.351 & ++---- & 0.008 & 0.028 & 0.771 & +-+-+- \\
\hline rs7279487 & $\mathrm{T}$ & C & C & 0.033 & 0.041 & 0.414 & -+-+++ & 0.008 & 0.040 & 0.848 & +++--- \\
\hline rs844996 & $\mathrm{T}$ & C & $\mathrm{G}(\mathrm{C})$ & -0.001 & 0.049 & 0.977 & +++-++ & 0.063 & 0.048 & 0.188 & +++-++ \\
\hline
\end{tabular}

Abbreviations: EA, effect allele; OA, other allele; MA, minor allele from Table 1 to enable comparison (alleles within parentheses are to clarify strand differences); SE, standard error.

$P$-values $<0.05$ are highlighted in bold. Dir is the direction of effect of LBC1921, ABC1936, Manchester males, Manchester females, Newcastle males and Newcastle females.

non-human primate marmoset. ${ }^{46}$ SYNJ2 is differentially expressed in major depressive disorder, ${ }^{47}$ and is part of the neuronal processes implicated in cognitive function ${ }^{48}$ and cognitive deficits, ${ }^{49}$ is associated with age-related spatial learning impairments in rats, ${ }^{50}$ and has been hypothesised to be involved in normal development of the brain. ${ }^{49}$ SYNJ2 is a target for the 'Genes to Cognition' project (http://www.genes2cognition.org). Alternative splicing of SYNJ2 results in multiple transcript variants and is an important candidate considering its role in ubiquitous signal-transduction pathways. ${ }^{51}$ Gene Ontology (GO) links five biological processes to SYNJ2 through inferred electronic annotation (brain development (GO: 007420), dephosphorylation (GO: 0016311), inositol phosphate dephosphorylation (GO: 0046855), phosphoinositide dephosphorylation (GO: 0046856) and intracellular distribution of mitochondria (GO: 0048312)).

A limitation of our study is the lack of consistent association between cohorts, both at the SNP level, despite using the same SNPs, and at the phenotype level, despite each general factor and intelligence test capturing overlapping cognitive abilities. There are many reasons that may explain this common occurrence in replication studies. First, and most likely, is that the original finding in LBC1936 may be a false positive as none of the associations reported here surpassed the Bonferroni correction. Second, the result may be a true positive with overestimated effect sizes, which would reduce the power of detection in our replication cohorts. ${ }^{52}$ Third, the original finding may be a true positive and the effect size unbiased, but sample size, selection bias, presence of allelic heterogeneity or hidden population sub-structure, and phenotypic heterogeneity might prevent replication. The cohorts differ in time of recruitment, age, location and cognitive tests as detailed in the Materials and Methods section. Generally, those samples that have a greater number of more varied cognitive tests will have a more reliable general cognitive ability phenotype, and the same principle applies within cognitive domains. These differences may have limited the degree to which the attempted replication was truly a replication. The lack of association in the Manchester sample may be due to subtle population substructure differences between North East and North West England; however, there is no strong evidence of population stratification, as reported previously. ${ }^{26}$ However, the lack of association in the Manchester sample should not be due to phenotypic heterogeneity as the cognitive tasks were performed by the same test co-ordinators and the collection of tests were the same for both Newcastle and Manchester cohorts. Furthermore, the non-replication of specific SNPs could be explained by varying linkage disequilibrium in the different cohorts between the causative variant (presumably not genotyped) and the tagging SNPs. Another limitation of the study was that the SNP selection strategy was based on tagging the genes with HapMap SNPs. The coverage of two genes, FRAP1 and MAT2A, with HapMap SNPs was small. 
Therefore, these genes cannot be definitely excluded as associated with cognitive abilities.

This is the first survey of longevity genes, unveiled by a comparative genomics approach, for association with cognitive abilities across the life course. We are the first to report SYNJ2, as a preliminary candidate gene awaiting independent replication, influencing cognitive abilities.

\section{CONFLICT OF INTEREST}

The authors declare no conflict of interest.

\section{ACKNOWLEDGEMENTS}

We thank the cohort participants and team members who contributed to these studies. Genotyping of the CAGES cohorts and the analyses conducted here were supported by the UK's Biotechnology and Biological Sciences Research Council (BBSRC). Phenotype collection in the Lothian Birth Cohort 1936 was supported by Research Into Ageing (continues as part of Age UK's The Disconnected Mind project). Phenotype collection in the Lothian Birth Cohort 1921 was supported by the BBSRC, The Royal Society and The Chief Scientist Office of the Scottish Government. Phenotype collection in the Aberdeen Birth Cohort 1936 was supported by BBSRC, the Welcome Trust and the Alzheimer's Research trust. Phenotype collection in the Manchester and Newcastle Longitudinal Studies of Cognitive Aging cohorts was supported by Social Science Research Council, Medical Research Council, Economic and Social Research Council, Research Into Ageing, Wellcome Trust and Unilever plc. LML is supported by AXA Research Fund during some of this work. ML is a Royal Society of Edinburgh/Lloyds TSB Foundation for Scotland Personal Research Fellow. The work was undertaken in The University of Edinburgh Centre for Cognitive Ageing and Cognitive Epidemiology, part of the cross council Lifelong Health and Wellbeing Initiative (G0700704/84698). Funding from the BBSRC, EPSRC, ESRC and MRC is gratefully acknowledged.

\section{AUTHOR CONTRIBUTIONS}

Data analysis: Lopez. Study design: Lopez and Deary. Manuscript Preparation: Lopez, Deary, Harris, Luciano, Gow, Starr and Pendleton. Individual study design/management: Tenesa, Payton, Ke, Whalley, Fox, Ollier, Pickles, Porteous, Horan, Pendleton, Starr and Deary. Genetic preparation: Lopez, Liewald, Davies, Harris, Luciano and Tenesa. Phenotype preparation: Lopez, Luciano, Gow, Payton, Ke, Whalley, Fox, Ollier, Pickles, Horan, Pendleton, Starr and Deary. Manuscript review: Lopez, Harris, Luciano, Liewald, Davies, Gow, Tenesa, Payton, Ke, Whalley, Fox, Ollier, Pickles, Porteous, Horan, Pendleton, Starr and Deary.

1 Ho L: Ageing: Scientific Aspects. London, UK, 2005

2 Melzer D, McWilliams B, Brayne C, Johnson T, Bond J: Profile of disability in elderly people: estimates from a longitudinal population study. BMJ 1999; 318: 1108-1111.

3 Deary IJ: Individual differences in cognition: British contributions over a century. $\mathrm{Br} \mathrm{J}$ Psychol 2001; 92: 217-237.

4 Wilson RS, Beckett LA, Barnes LL et al: Individual differences in rates of change in cognitive abilities of older persons. Psychol Aging 2002; 17: 179-193.

5 Deary IJ, Corley J, Gow AJ et al: Age-associated cognitive decline. Br Med Bull 2009; 92: $135-152$.

6 Fillit HM, Butler RN, O'Connell AW et al: Achieving and maintaining cognitive vitality with aging. Mayo Clin Proc 2002; 77: 681-696.

7 Deary IJ, Johnson W, Houlihan LM: Genetic foundations of human intelligence. Hum Genet 2009; 126: 215-232.

8 Calvin CM, Deary IJ, Fenton C et al: Intelligence in youth and all-cause-mortality: systematic review with meta-analysis. Int J Epidemiol 2011; 40: 626-644.

9 Der G, Batty GD, Deary IJ: The association between IQ in adolescence and a range of health outcomes at 40 in the 1979 US National Longitudinal Study of Youth. Intelligence 2009; 37: 573-580.

10 Christensen H, Mackinnon AJ, Korten A, Jorm AF: The 'common cause hypothesis' of cognitive aging: evidence for not only a common factor but also specific associations of age with vision and grip strength in a cross-sectional analysis. Psychol Aging 2001; 16: 588-599.

11 Maier H, McGue M, Vaupel JW, Christensen K: Cognitive Impairment and Survival at Older Ages. Berlin: Springer, 2003.

12 Batty GD, Deary IJ, Gottfredson LS: Premorbid (early life) IQ and later mortality risk: systematic review. Ann Epidemiol 2007; 17: 278-288.
13 Deary I: Why do intelligent people live longer? Nature 2008; 456: 175-176.

14 Mitchell BD, Hsueh WC, King TM et al: Heritability of life span in the Old Order Amish. Am J Med Genet 2001; 102: 346-352.

15 Kirkwood TB, Feder M, Finch CE et al: What accounts for the wide variation in life span of genetically identical organisms reared in a constant environment? Mech Ageing Dev 2005; 126: 439-443.

16 Partridge L: The new biology of ageing. Philos Trans R Soc Lond Ser B 2010; 365 : 147-154.

17 Smith ED, Tsuchiya M, Fox LA et al: Quantitative evidence for conserved longevity pathways between divergent eukaryotic species. Genome Res 2008; 18: 564-570.

18 Shmueli O, Horn-Saban S, Chalifa-Caspi V et al: GeneNote: whole genome expression profiles in normal human tissues. C R Biol 2003; 326: 1067-1072.

19 Deary IJ, Whiteman MC, Starr JM, Whalley LJ, Fox HC: The impact of childhood intelligence on later life: following up the Scottish mental surveys of 1932 and 1947. J Pers Soc Psychol 2004; 86: 130-147.

20 Deary IJ, Whalley LJ, Starr JM: A Lifetime of Intelligence: Follow-up Studies of the Scottish Mental Surveys of 1932 and 1947. Washington, DC: American Psychological Association, 2009.

21 Rabbitt PMA, McInnes L, Diggle P et al: The University of Manchester longitudinal study of cognition in normal healthy old age, 1983 through 2003. Aging Neuropsychol Cogn 2004; 11: 245-279.

22 Scottish Council for Research in Education: Mental Survey Committee; in Thomson GH (ed): Scottish Council for Research in Education: The Trend of Scottish Intelligence: A Comparison of the 1947 and 1932 Surveys of the Intelligence of Eleven-Year-Old Pupils. London: University of London Press, 1949.

23 Deary IJ, Gow AJ, Taylor MD et al: The Lothian Birth Cohort 1936: a study to examine influences on cognitive ageing from age 11 to age 70 and beyond. BMC Geriatr 2007; 7: 28.

24 Folstein MF, Folstein SE, McHugh PR: 'Mini-mental state'. A practical method for grading the cognitive state of patients for the clinician. J Psychiatr Res 1975; 12: 189-198.

25 Anderson TM, Sachdev PS, Brodaty H, Trollor JN, Andrews G: Effects of sociodemographic and health variables on Mini-Mental State Exam scores in older Australians. Am J Geriatr Psychiatry 2007; 15: 467-476.

26 Davies G, Tenesa A, Payton A et al: Genome-wide association studies establish that human intelligence is highly heritable and polygenic. Mol Psychiatry 2011; 16: 996-1005.

27 Wechsler D: WMS-III: Wechsler Memory Scale Administration and Scoring Manual. Psychological Corp (1997) London, UK, 1998.

28 Deary IJ, Der G, Ford G: Reaction times and intelligence differences - a populationbased cohort study. Intelligence 2001; 29: 389-399.

29 de Bakker PI, Yelensky R, Pe'er I, Gabriel SB, Daly MJ, Altshuler D: Efficiency and power in genetic association studies. Nat Genet 2005; 37: 1217-1223.

30 Barrett JC, Fry B, Maller J, Daly MJ: Haploview: analysis and visualization of LD and haplotype maps. Bioinformatics 2005; 21: 263-265.

$31 \mathrm{Li} \mathrm{Y,} \mathrm{Willer} \mathrm{CJ,} \mathrm{Ding} \mathrm{J,} \mathrm{Scheet} \mathrm{P,} \mathrm{Abecasis} \mathrm{GR:} \mathrm{MaCH}$ : using sequence and genotype data to estimate haplotypes and unobserved genotypes. Genet Epidemiol 2010; 34: 816-834.

32 Luciano M, Hansell N, Lahti J et al: Whole genome association scan for genetic polymorphisms influencing information processing speed. Biol Psychol 2011; 86: 193-202.

33 Houlihan LM, Davies G, Tenesa A et al: Common variants of large effect in F12, KNG1, and HRG are associated with activated partial thromboplastin time. Am J Hum Genet 2010; 86: 626-631.

34 Luciano M, Gow AJ, Hayward C et al: Cognitive aging from age 11 to 70 years, information processing speed, and APOE variation: the Lothian Birth Cohort 1936 Study. Psychol Aging 2009; 24: 129-138.

35 Houlihan LM, Wyatt ND, Harris SE et al: Variation in the uric acid transporter gene (SLC2A9) and memory performance. Hum Mol Genet 2010; 19: 2321-2330.

36 Houlihan LM, Harris SE, Luciano M et al: Replication study of candidate genes for cognitive abilities: the Lothian Birth Cohort 1936. Genes Brain Behav 2009; 8: 238-247.

37 Johnson W, te Nijenhuis J, Bouchard TJ: Still just $1 \mathrm{~g}$ : consistent results from five test batteries. Intelligence 2008; 36: 81-95.

38 Purcell S, Neale B, Todd-Brown K et al: PLINK: a tool set for whole-genome association and population-based linkage analyses. Am J Hum Genet 2007; 81: 559-575.

39 Li Y, Willer C, Sanna S, Abecasis G: Genotype imputation. Annu Rev Genom Hum Genet 2009; 10: 387-406.

40 Willer CJ, Li Y, Abecasis GR: METAL: fast and efficient meta-analysis of genomewide association scans. Bioinformatics 2010; 26: 2190-2191.

41 Shen Q, Zhang Z, Yu L et al: Common variants near TERC are associated with leukocyte telomere length in the Chinese Han population. Eur J Hum Genet 2011; 19: 721-723.

42 Perneger TV: What's wrong with Bonferroni adjustments. BMJ 1998; 316: 1236-1238.

43 Nyholt DR: A simple correction for multiple testing for single-nucleotide polymorphisms in linkage disequilibrium with each other. Am J Hum Genet 2004; 74 : 765-769.

44 Purcell S, Cherny SS, Sham PC: Genetic Power Calculator: design of linkage and association genetic mapping studies of complex traits. Bioinformatics 2003; 19: 149-150. 
45 Nemoto $Y$, Wenk MR, Watanabe $M$ et al: Identification and characterization of a synaptojanin 2 splice isoform predominantly expressed in nerve terminals. $\mathrm{J} \mathrm{Biol} \mathrm{Chem}$ 2001; 276: 41133-41142.

46 Datson NA, Morsink MC, Steenbergen PJ et al: A molecular blueprint of gene expression in hippocampal subregions CA1, CA3, and DG is conserved in the brain of the common marmoset. Hippocampus 2009; 19: 739-752.

47 Aston C, Jiang L, Sokolov BP: Transcriptional profiling reveals evidence for signaling and oligodendroglial abnormalities in the temporal cortex from patients with major depressive disorder. Mol Psychiatry 2005; 10: 309-322.

48 Laumonnier F, Cuthbert PC, Grant SG: The role of neuronal complexes in human X-linked brain diseases. Am J Hum Genet 2007; 80: 205-220.
49 Nagamani SC, Erez A, Eng C et al: Interstitial deletion of 6q25.2-q25.3: a novel microdeletion syndrome associated with microcephaly, developmental delay, dysmorphic features and hearing loss. Eur J Hum Genet 2009; 17: 573-581.

50 Burger C, Lopez MC, Feller JA, Baker HV, Muzyczka N, Mandel RJ: Changes in transcription within the CA1 field of the hippocampus are associated with age-related spatial learning impairments. Neurobiol Learn Mem 2007; 87: 21-41.

51 Nemoto Y, Arribas M, Haffner C, DeCamilli P: Synaptojanin 2, a novel synaptojanin isoform with a distinct targeting domain and expression pattern. J Biol Chem 1997; 272: 30817-30821.

52 Zollner S, Pritchard JK: Overcoming the winner's curse: estimating penetrance parameters from case-control data. Am J Hum Genet 2007; 80: 605-615.

Supplementary Information accompanies the paper on European Journal of Human Genetics website (http://www.nature.com/ejhg) 